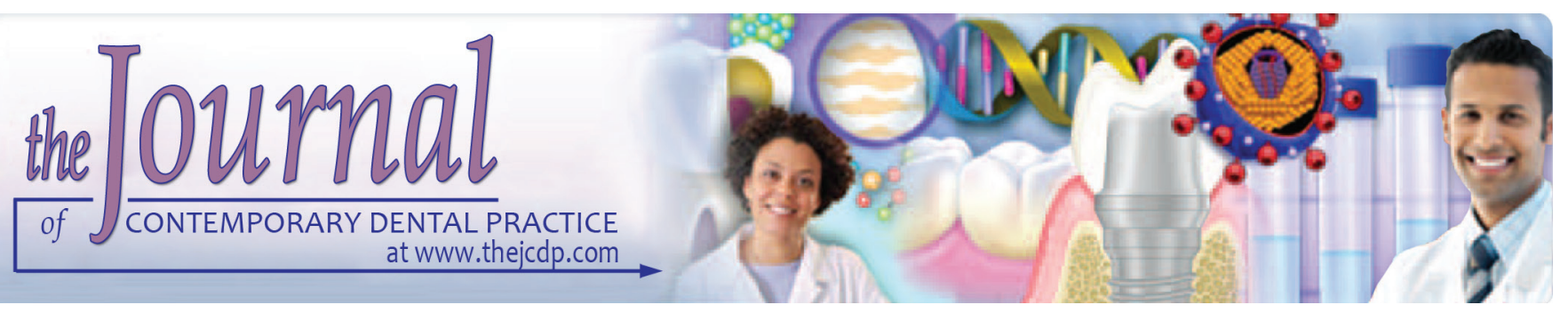

\title{
Comparative Evaluation of the Discoloration of Microhybrid and Nanohybrid Composite Resins by Different Beverages: A Spectrophotometric Analysis
}

\author{
${ }^{1}$ Rohini P Mahajan, ${ }^{2}$ Vanitha U Shenoy, ${ }^{3}$ Sumanthini MV, ${ }^{4}$ Harsha P Mahajan, ${ }^{5}$ Pranjal S Walzade, ${ }^{6}$ Rajan Mangrolia
}

\begin{abstract}
Background: Composite resins are choice of restorations due to their lifelike characteristics. Recently "microhybrid "and "nanohybrid" composites have been introduced. Microhybrids and Nanocomposites have a small particle size and possess better qualities as compared to traditional composite resins. The intake of beverages like black tea, coffee green tea has increased in the community. The beverages cause discoloration with time on the composite restorations making it aesthetically unacceptable.

Aim: Aim is to evaluate discoloration of microhybrid and nanohybrid composite resins by black tea, black coffee, green tea and distilled water at $0,3,6,9$, and 12 days.

Methodology: 160 samples were fabricated in molds from two nanohybrid composite and two microhybrid composites. They were light cured and allocated into four groups were stored in an incubator throughout the experiment at $37^{\circ} \mathrm{C}$. Initial baseline color was recorded by a spectrophotometer. These samples were immersed in vials containing black tea, black coffee, green tea and distilled water for three, six, nine, 12 days. Color analysis was done at each interval.
\end{abstract}

Statistical analysis: Data was tabulated and the readings after immersion and baseline were compared between four composite resins. Data were analyzed using the appropriate test by testing normality. A $p$ value $<0.05$ was considered to be significant.

1-3,5 Department of Conservative Dentistry and Endodontics, MGM Dental College and Hospital, Navi Mumbai, Maharashtra, India

${ }^{4}$ Department of Dentistry, Rajiv Gandhi Medical College, Kalwa, Thane, Maharashtra, India

${ }^{6}$ Department of Conservative Dentistry and Endodontics, SMBT Dental College, Sangamner, Ahmednagar, Maharashtra, India

Corresponding Author: Rohini P Mahajan, Department of Conservative Dentistry and Endodontics, MGM Dental College and Hospital, Navi Mumbai, Maharashtra, India, Phone: +918600167260, e-mail: rohinimahajan545@gmail.com
Results: Significant discoloration was observed in all four composite resins. Discoloration increased from the third day to 12th day. Maximum discoloration was observed on the 12th day.

Conclusion: The order of discoloration in the beverages was: Flash $>$ Tetric $n$ ceram $>$ matrix $>$ tetricceram. The order of discoloration caused by test solutions on composite resin was: Black coffee $>$ black tea $>$ green tea $>$ distilled water.

Keywords: Discoloration, Microhybrid, and nanohybrid composite resins, Spectrophotometer, Test solutions.

How to cite this article: Mahajan RP, Shenoy VU, Sumanthini MV, Mahajan HP, Walzade PS, Mangrolia R. Comparative Evaluation of the Discoloration of Microhybrid and Nanohybrid Composite Resins by Different Beverages: A Spectrophotometric Analysis. J Contemp Dent Pract 2019;20(2):226-230.

Source of support: Nil

Conflict of interest: None

\section{INTRODUCTION}

Color stability of composite resin (CR) restoration is important not only at the initial stage of the restoration but also over a longer period of time. The susceptibility to staining of tooth-colored restorations has been attributed to internal and external factors. Factors include the material itself, environment, patient and clinician. The composition of the matrix, amount of filler loading, filler size, along with the biphasic nature of CR are intrinsic components to consider. The type of staining agent, the duration of exposure, and its compatibility with the matrix are factors influencing the susceptibility to staining. The patient's diet and oral hygiene habits, along with the clinician's manipulation of the material important as well. ${ }^{1}$

Recently, nanohybrid (NH) and microhybrid (MH) CR have been introduced that have nanoparticles measuring about 25 nanometers and having about $79.5 \%$ filler. Since particles have become smaller, these CR are expected 
to be more polishable and less discolored following absorption of pigments. This technology also claims that it has provided suitable mechanical properties for anterior and posterior restorations and it has less shrinkage and leakage. $^{2}$

Intake of black tea, black coffee, green tea has become popular in society. Studies have shown that $\mathrm{MH}$ and $\mathrm{NH}$ $\mathrm{CR}$ are susceptible to color alteration when exposed to staining solutions like coffee, cola, tea and wine. ${ }^{3}$

Evaluation of the discoloration of esthetic materials has been done by several color systems that are used for describing color parameters among which, the CIE and Munsell color systems have the highest application in studies. Color change at $\Delta \mathrm{E}>3.3$ is clinically perceptible. ${ }^{4}$

Color stability of various $\mathrm{MH}, \mathrm{NH} \mathrm{CR}$ in different beverages have been studied, wherein the color and shade changes were noted the most in coffee and tea ${ }^{5}$ whereas, some authors observed coffee to have the most effect in color change in the samples tested. ${ }^{6}$ There are many newly introduced NHCR in the market and few studies regarding the color stability of these in the literature. Thus, the aim of this study is to evaluate discoloration of MH and NHCR by black tea, black coffee, green tea and distilled water at $0,3,6,9,12$ days. The null hypothesis tested was there is no difference in the discoloration of $\mathrm{MH}$ and $\mathrm{NH} \mathrm{CR}$ by different beverages at various time intervals.

\section{MATERIALS AND METHODS}

Two MH (tetric ceram and matrix) and Two NH CR materials; tetric $\mathrm{N}$ ceram (Ivoclar Vivadent India) and Flash (Medicept Dental, India)], composition, as shown in Table 1, were selected and discoloration of the materials was tested in black tea, black coffee, green tea, and distilled water.

For the preparation of solutions tea (Tajmahal, Brookbond, WestBengal, India), coffee (Bru, Karnataka, India), green tea (Lipton, Haryana, India) and distilled water were used.

For the preparation of samples, a clean glass slab was kept on a flat surface and a Teflon mold was placed over it. CR was dispensed and placed into the mold with help of
CR filling instrument. A Mylar strip was placed beneath and over the CR and a glass plate was placed on mylar strip to compress it.

The CR in the mold was light-cured of both sides as per manufacturer's instruction using a halogen light curing unit (Guilin Woodpecker Medical Instrument Co Ltd), removed from the mold and polished circumferentially using CR polishing kit (Shofu dental corporation, Japan). A mark was made on one surface of the CR sample and the other surface with mylar finish was used for color analysis throughout the study.

Likewise, 160 samples measuring $10 \mathrm{~mm} \times 2 \mathrm{~mm}$ dimension were prepared from $\mathrm{MH}$ and NHCR (80 in each group) of shade A2 enamel. They were further divided into two sub groups of 40 each to be immersed in test solutions. Initial color analysis was done with a spectrophotometer (Datacolor 650) and values recorded.

For the preparation of beverages $200 \mathrm{~mL}$ of water was boiled and 2 prefabricated bags of tea or green tea were placed in boiling water and then cooled to room temperature. For the preparation of black coffee solution $4 \mathrm{~g}$ of coffee, the powder was measured added to $200 \mathrm{~mL}$ boiling water and cooled to room temperature. After cooling $\mathrm{pH}$ of test solutions were recorded. $10 \mathrm{~mL}$ of test solution was transferred in fresh vials and samples were immersed in these vials. The fresh solution was prepared at each interval, the procedure repeated as above and color analysis was done at 3, 6, 9, 12 days.

\section{Statistical Analysis}

The results of the discoloration were calculated on the basis of $\Delta \mathrm{E}$ which is the difference between $\mathrm{L}^{*} \mathrm{a}^{*} \mathrm{~b}^{*}$ coordinates. It was calculated as was calculated as $\Delta \mathrm{E}=$ $\left[\Delta \mathrm{L}^{2}+\Delta \mathrm{a}^{2}+\Delta \mathrm{b}^{2}\right]^{1 / 2}$

The data obtained was subjected to statistical analysis using SPSS software version 20 and results tabulated. The normality of data was tested by using ANOVA for day wise comparison. Mauchly's test of sphericity, Kolmogorov-Smirnov test and Shapiro-Wilk, Greenhouse-Geisser and Tukey's post-hoc multiple

Table 1: Composition of the four composite resins

\begin{tabular}{|c|c|c|c|}
\hline Category & Composition & $\begin{array}{l}\text { Mean Filler } \\
\text { size }(\mu m)\end{array}$ & Manufacturer \\
\hline Microhybrid & $\begin{array}{l}\text { Bis-GMA, UDMA, barium glass filler, Ytterbium trifluoride, mixed oxide, } \\
\text { highly dispersed silica, prepolymers, additives, catalyst, and stabilizers }\end{array}$ & 0.6 & $\begin{array}{l}\text { Ivoclarvivadent, Andheri, } \\
\text { Mumbai }\end{array}$ \\
\hline Microhybrid & $\begin{array}{l}\text { Bis-GMA, UDMA, barium glass filler, Ytterbium trifluoride, mixed oxide, } \\
\text { highly dispersed silica, prepolymers, additives, catalyst, and stabilizers }\end{array}$ & 0.6 & Densply, Noida, India \\
\hline Nanohybrid & $\begin{array}{l}\text { (BISGMA), (UDMA) or Barium glass (PEX), and an inorganic filler } \\
\text { such as silicon dioxide (silica) an inorganic filler such as silicon dioxide } \\
\text { (silica) }\end{array}$ & 0.4 & $\begin{array}{l}\text { Ivoclar vivadent, Andheri, } \\
\text { Mumbai }\end{array}$ \\
\hline Nanohybrid & $\begin{array}{l}\text { (BISGMA), (UDMA) or, barium glass, (PEX), an inorganic filler silicon } \\
\text { dioxide }\end{array}$ & 0.4 & $\begin{array}{l}\text { Medicept, Mulund, } \\
\text { Mumbai }\end{array}$ \\
\hline
\end{tabular}


comparison tests were performed. A $p<0.05$ was considered to be significant.

\section{RESULTS (TABLE 2)}

In the present study, it was observed in group 1 (Graph 1A): Tetric Ceram gave acceptable results in distilled water at all time intervals and at day 3 in coffee and day 3 and 6 in green tea $\Delta \mathrm{E}^{*}{ }_{\mathrm{ab}}$ was $<3.3$, rest of the results gave an $\Delta \mathrm{E}_{\mathrm{ab}}^{*}$ which was $>3.3$

In group 2 (Graph 1B) matrix gave acceptable results in distilled water at all time intervals, at day 3 in black tea and day 3, 6, 9 in green tea. $\Delta \mathrm{E}_{\mathrm{ab}}^{*}$ was $<3.3$.

In group 3 (Graph 1C): Tetric N Ceram gave acceptable results in distilled water and green tea at all time intervals and at day 3 in black tea $\Delta \mathrm{E}^{*}{ }_{\mathrm{ab}}$ was $<3.3$.

In group 4 (Graph 1D): flash gave acceptable results in distilled water at all time intervals and at day 3 for black tea and day $3,6,9 \Delta \mathrm{E}_{\mathrm{ab}}^{*}$ was $<3.3$. Rest of the results gave $\Delta \mathrm{E}_{\mathrm{ab}}^{*}$ which was $>3.3$.

On comparing the discoloration caused at various time intervals by the test solutions on all the tested $\mathrm{CR}$, the $p$ value was $>0.05$ except it in MHCR-tetric N Ceram group 1 in distilled water it was not statistically significant $(p>0.05)$.

\section{DISCUSSION}

$\mathrm{MH}$ and NHCR used as aesthetic materials give a good finish and polish were chosen for the present study.
The discoloration of CR may be attributed to an intrinsic factor which involves physiochemical alteration within the material. Extrinsic staining involves surface discoloration by extrinsic substances. Research has found that beverages including coffee, tea caused significant color changes in CR. ${ }^{7}$

In the current study, CR samples were immersed in black coffee and black tea exhibited discoloration which could be due to deposition of tannins found in tea, coffee that causes brown stains. However green tea caused less staining than black coffee and black tea as the colorants present in green tea is not as effective as that present in black tea and coffee. The darker the coffee, the more it stains the teeth. ${ }^{8}$

Uzun et al. in $2015^{8}$ evaluated the color change of $\mathrm{NH}$ and MHCR by distilled water, tea, coffee, etc. for 7 days and observed an $\Delta \mathrm{E}=5.90$ in distilled water, in coffee $\Delta \mathrm{E}=4.61$ and tea $\Delta \mathrm{E}=4.84$. In the present study at the 6th-day interval $\Delta \mathrm{E}=1.04,8.41$ and 3.38, respectively for black coffee and black tea. This could be due to the influence of resin monomers on the color stability of CR resins which are associated with the hydrophobicity and water absorption properties of the resin monomers. ${ }^{9}$ According to Ferracane ${ }^{10}$ greater the volume of filler particles in the CR composition, lower is the conversion degree.

The presence of microcracks in the resin matrix as a result of swelling and plasticizing effects, along with interfacial gaps created between the filler and resin

Table 2: Daywise and beverage comparison for discoloration in the four CR

\begin{tabular}{|c|c|c|c|c|c|c|c|c|c|c|}
\hline \multirow[b]{3}{*}{$C R$} & \multirow[b]{3}{*}{ Time interval } & \multicolumn{8}{|c|}{$\Delta E$ values } & \multirow[b]{3}{*}{$p$ value } \\
\hline & & \multicolumn{2}{|c|}{$D W$} & \multicolumn{2}{|c|}{ Coffee } & \multicolumn{2}{|c|}{ Tea } & \multicolumn{2}{|c|}{ Green tea } & \\
\hline & & Mean & $S D$ & Mean & $S D$ & Mean & $S D$ & Mean & $S D$ & \\
\hline \multirow{5}{*}{$\begin{array}{l}\text { Tetric } \\
\text { Ceram } \\
(\mathrm{MH})\end{array}$} & Day 3 & 0.80 & 0.67 & 2.45 & 0.88 & 3.56 & 1.11 & 1.16 & 0.48 & 0.000 \\
\hline & Day 6 & 0.90 & 0.51 & 3.83 & 1.83 & 6.60 & 1.47 & 2.24 & 0.43 & 0.000 \\
\hline & Day 9 & 1.00 & 0.60 & 6.52 & 2.28 & 9.83 & 1.91 & 3.63 & 1.16 & 0.000 \\
\hline & Day 12 & 1.02 & 0.58 & 10.62 & 2.33 & 12.86 & 2.43 & 4.15 & 1.19 & 0.000 \\
\hline & $p$ value & & & & & & & & & \\
\hline \multicolumn{11}{|l|}{ Matrix } \\
\hline \multirow[t]{5}{*}{$(\mathrm{MH})$} & Day 3 & 0.11 & 0.15 & 3.80 & 2.48 & 2.63 & 2.29 & 0.77 & 0.42 & 0.000 \\
\hline & Day 6 & 0.48 & 0.54 & 6.60 & 3.37 & 3.98 & 2.65 & 1.68 & 0.60 & 0.000 \\
\hline & Day 9 & 0.85 & 0.55 & 10.68 & 3.54 & 6.55 & 3.42 & 2.78 & 1.01 & 0.000 \\
\hline & Day 12 & 1.06 & 0.44 & 13.84 & 3.47 & 9.94 & 3.72 & 3.54 & 0.89 & 0.000 \\
\hline & $p$ value & \multicolumn{2}{|c|}{0.000} & \multicolumn{2}{|c|}{0.000} & \multicolumn{2}{|c|}{0.000} & \multicolumn{2}{|c|}{0.000} & \\
\hline \multirow{5}{*}{$\begin{array}{l}\text { Tetric n } \\
\text { Ceram } \\
(\mathrm{NH})\end{array}$} & Day 3 & 0.10 & 0.02 & 5.68 & 1.49 & 2.42 & 0.78 & 0.64 & 0.31 & 0.000 \\
\hline & Day 6 & 1.04 & 0.59 & 8.41 & 2.11 & 3.38 & 0.59 & 0.94 & 0.55 & 0.000 \\
\hline & Day 9 & 1.99 & 0.66 & 11.66 & 2.01 & 4.97 & 0.88 & 1.43 & 0.57 & 0.000 \\
\hline & Day 12 & 2.62 & 0.89 & 14.72 & 1.68 & 6.20 & 0.94 & 2.17 & 0.95 & 0.000 \\
\hline & $p$-value & \multicolumn{2}{|c|}{0.000} & \multicolumn{2}{|c|}{0.000} & \multicolumn{2}{|c|}{0.000} & \multicolumn{2}{|c|}{0.000} & \\
\hline \multirow{5}{*}{$\begin{array}{l}\text { Flash } \\
(\mathrm{NH})\end{array}$} & Day 3 & 0.70 & 0.30 & 3.90 & 1.00 & 1.57 & 0.44 & 1.31 & 0.32 & 0.000 \\
\hline & Day 6 & 0.98 & 0.35 & 8.30 & 1.46 & 3.48 & 0.40 & 2.15 & 09.61 & 0.000 \\
\hline & Day 9 & 1.26 & 0.51 & 12.28 & 1.75 & 5.71 & 1.33 & 3.26 & 0.76 & 0.000 \\
\hline & Day 12 & 1.53 & 0.76 & 15.04 & 1.23 & 8.62 & 1.22 & 3.99 & 0.86 & 0.000 \\
\hline & $p$ value & \multicolumn{2}{|c|}{0.003} & \multicolumn{2}{|c|}{0.000} & \multicolumn{2}{|c|}{0.000} & \multicolumn{2}{|c|}{0.000} & \\
\hline
\end{tabular}



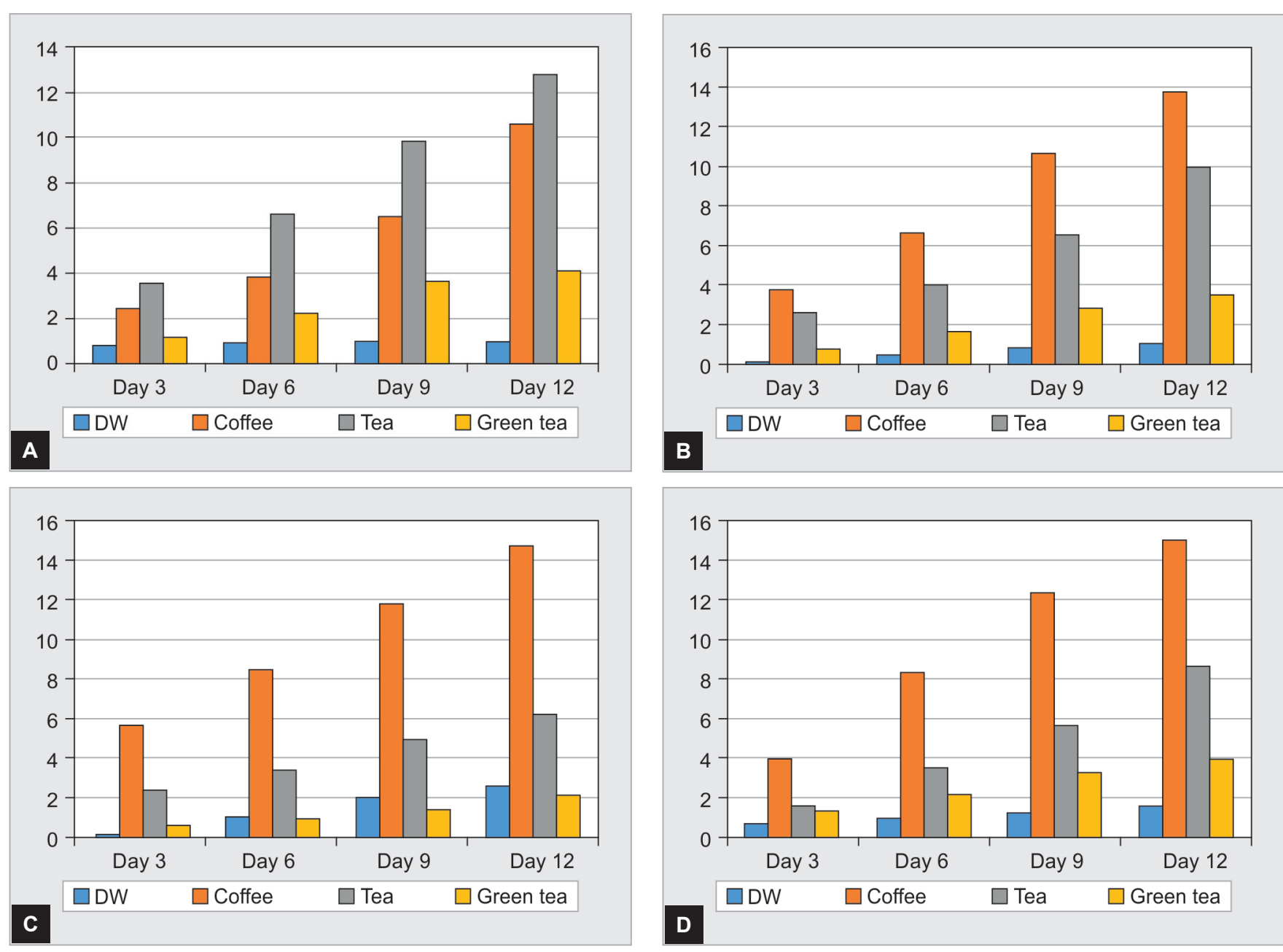

Graph $1 A$ to $D$ : Represents discoloration in form of $\Delta E$ in all the beverages at 3, 6, 9, and 12 days: (A) Tetric Ceram (Group 1); (B) Matrix (Group 2); (C) Tetric N Ceram (Group 3); (D) Flash (Group 4)

matrix allow stain penetration and discoloration of the restoration. ${ }^{11,12}$ The colorants polarity can determine its degree of CR penetration. Less polar colorants may be easily absorbed inside the material, whereas more polar colorants tend to be adsorbed on the surface of the material. ${ }^{9}$

In the present study all the CR tested had filler by volume in the range of $55-59 \%$ and filler particle size in the range of $0.4-0.6 \mu \mathrm{m}$, thus discoloration was observed in all the groups. Also, all the CR tested contained barium glass particles, studies conducted by Hubbezoglu et al. in $2008,{ }^{13}$ and Hirata et al. in $2000^{14}$ stated that CR with barium glass particles tends to absorb more water. These particles can thin light and thus reduce the luminosity. The presence of these particles in the test materials in the present study all $4 \mathrm{CR}$ is probably another reason for its significant discoloration. An extremely important part is also played by the coupling agent that, to some extent, protects the filler against degradation through hydrolysis. ${ }^{15}$

Mylar strip was kept above and below the samples during fabrication so that the $\mathrm{CR}$ is confined inside the mold also a flattened surface is obtained. ${ }^{16}$
The quartz-tungsten-halogen light-curing unit was used in this study which had a tip diameter of $12 \mathrm{~mm}$, the intensity of $450 \mathrm{~mW} / \mathrm{cm}^{2}$ and wavelength between $400 \mathrm{~nm}$ and $500 \mathrm{~nm}$ which is sufficient to cure CR restoration directly to a depth of $2 \mathrm{~mm}$. Specimen diameter was $10 \mathrm{~mm}$ which was less than the tip diameter of the light curing unit tip (12 mm) to ensure the complete surface of the sample gets cured. ${ }^{17}$

In the current study, all the CR samples were immersed in the test solutions for a period of 3, 6, 9 and 12 days. This time interval was chosen assuming an average person drinks 2-3 cups of coffee every day with the mean duration of drinking 1 cup of coffee is 15 minutes, thus a 30-day storage time is approximately equal to 30 months of coffee consumption. ${ }^{18}$ Thus every 24 hours of immersion in beverage corresponds to 1 month of consumption of beverage likewise 3 days correspond to 3 months, 6 days correspond to 6 months, 9 days correspond to 9 months and 12 days corresponds to 12 month time interval.

Teflon mold was used in this study for the fabrication of samples as it does not adhere to the mold. As in a clinical situation, once the restoration has been completed, 
it will be finished and polished. In the present study, the CR samples were finished to remove the excess material/ irregularities on its circumference.The lower surface of the sample was left untouched by the finishing and polishing procedure. They had only the Mylar finish to avoid the differences in surface finish and to avoid bias in the results.

Samples were placed in an incubator during the study to facilitate a constant environment at $37^{\circ} \mathrm{C}$ and in the dark such that light did not affect the CR.

The evaluation of the discoloration of the CR samples was carried out using the spectrophotometer which can detect color changes, making spectrophotometers a superior choice for accurate, repeatable color measurement. ${ }^{4}$ Hence, they were used in this study for evaluation of discoloration.

Limitations of the current study are, only a mylar finish was obtained, and discoloration was evaluated. The samples were immersed in test solutions throughout the entire time period. The effect of thermocycling on discoloration was not considered. Clinical evaluation of the discoloration of the newer generation CR needs to be carried out.

The null hypothesis was not accepted as there was a significant discoloration observed in all the 4 CRs in all the test solutions at the four-time intervals $(p>0.05)$.

\section{CONCLUSION}

Within the limitations and the conditions of the present study it can be concluded:

- Among the CR tested, the order of discoloration by the beverages was:

- Flash > Tetric N Ceram > Matrix > Tetric Ceram Among the beverages tested the order of discoloration of the $C R$ was :

- Black coffee $>$ Black tea $>$ Green tea $>$ Distilled water.

\section{REFERENCES}

1. Sarafianou A, Iosifidou S, Papadopoulos T, Eliades G. Color stability and degree of cure of direct composite restoratives after accelerated aging. Oper Dent 2007; 32(4):406-411.

2. Nasoohi N, Hoorizad M, Torabzadetari N. Effect of tea and coffee on color change of two type's CRs: Nanofilled and Micro hybrid. J Res Dent Sci 2011;7(4):18-22.

3. Nasim I, Neelakantan P, Sujeer R, Subbarao CV. Colour stability of microfilled, $\mathrm{MH}$ and nanoCRs-an in vitro study. J Dent 2010; 38(2):137-142.
4. CIE Publication No. 15.2 Colorimetry. Vienna: Central Bureau of the CIE; 1986:74.

5. Malekipour MR, Sharafi A, KazemiS, ShiraniF. Comparison of colour stability of a CR in different colour media. Dent Res J 2012;9:441-466.

6. Yannikakis SA, Zissis AJ, Polyzois GL, Caroni C.Colour stability of provisional resin restorative materials. J Prosthet Dent 1998; 80(5):533-539.

7. Tan BL, Yap AU, Ma HN, Chew J, Tan WJ. Effect of beverages on color and translucency of new tooth-colored restoratives. Oper Dent 2015; 40(2):56-65.

8. Uzun G, Keyf F, Burduroglu D. The effect of curing time and immersion solutions on discoloration of hybrid composites and nanocomposites. J Res Dent Sci 2015; 6:11-16.

9. Canay S, Hersek N, Tulunoglu I, Uzun G. Evaluation of colour and hardness changes of soft lining materials in food colourant solutions. J Oral Rehabil 1999; 26(10):821-829.

10. Ferracane JL. Hygroscopic and hydrolytic effects in dental polymer networks. Dent Mater 2006;22:211-222.

11. Bagheri R, Burrow MF, Tyas M. Influence of food simulating solutions and surface finish on susceptibility to staining of aesthetic restorative materials. J Dent 2005; 33: 389-398.

12. Aguiar FH, Georgetto MH, Soares GP, Catelan A, Dos Santos $\mathrm{PH}$, Ambrosano GM, Figueroba SR, Lovadino JR. Effect of different light-curing modes on degree of conversion, staining susceptibility and stain's retention using different beverages in a nanofilled CR. J Esthet Restor Dent 2011; 23(2):1 06-114.

13. Hubbezoglu I, Akaog lu B, Dogan A, Keskin S, Bolayir G, Ozc elik S, et al. Effect of bleaching on color change and refractive index of dental CRs. Dent Mater 2008; 27: 105-116.

14. Hirata R. Clinical alternative of laboratory CR systems when and how to use. J Brasileiro de Clinica \& Estéticaem Odontologia 2000;4:13-21.

15. Lovell LG, Lu H, Elliott JE, Stansbury JW, Bowman CN. The effect of cure rate on the mechanical properties of dental resins. Dent Mater 2001 Nov;17(6):504-511.

16. Singh S, Shah N, Mandlik J, Nair M, Jaggi S, Kanyal K. The effect of finishing and polishing procedures on the surface roughness of CR materials: an in-vitro study. IIOAB 2015; 6(3):17-21.

17. da Silva EM, Almeida GS, Poskus LT, Guimarães JG. Relationship between the degree of conversion, solubility and salivary sorption of a hybrid and a nanofilled resin composite. J Appl Oral Sci. 2008;16(2):161-166.

18. Zajkani E, AbdohTabrizi M, Ghasemi A, Torabzade H, Kharazifard MJ. Effect of Staining Solutions and Repolishing on Composites in Color Change. JIDA. 2013; 25(2): 83-90. 